Signal \& Image Processing : An International Journal (SIPIJ) Vol.8, No.4, August 2017

\title{
HYBRID DIFFUSION-STEERED MODEL FOR SUPPRESSING MULTIPLICATIVE NOISE IN ULTRASONOGRAMS
}

\author{
Suzan Kessy ${ }^{1}$, Baraka Maiseli ${ }^{2}$, and Michael Kisangiri ${ }^{1}$ \\ ${ }^{1}$ Department of Communication Science \& Engineering, Nelson Mandela African Institute \\ of Science \& Technology, Tanzania \\ ${ }^{2}$ Department of Electronics \& Telecommunication Engineering, \\ University of Dar-es- Salaam, Tanzania.
}

\begin{abstract}
Ultrasonograms refer to images generated through ultrasonography, a technique that applies ultrasound pulses to delineate internal structures of the body. Despite being useful in medicine, ultrasonograms usually suffer from multiplicative noises that may limit doctors to analyse and interpret them. Attempts to address the challenge have been made from previous works, but denoising ultrasonograms while preserving semantic features remains an open-ended problem. In this work, we have proposed a diffusion-steered model that gives an effective interplay between total variation and Perona-Malik models. Two parameters have been introduced into the framework to convexify our energy functional. Also, to deal with multiplicative noise, we have incorporated a log-based prior into the framework. Empirical results show that the proposed method generates sharper and detailed images. Even more importantly, our framework can be evolved over a longer time without smudging critical image features.
\end{abstract}

\section{KEYWORDS}

Denoising, ultrasound image, optimization, Perona-Malik, total variation

\section{INTRODUCTION}

Over the last 30 years, medical imaging has experienced a dramatic change: from X-rays that display internal body organs and tissues as shadows on a photographic film to computed tomography, magnetic resonance imaging, positron emission tomography, and ultrasound imaging (USI) that show cross-sectional images of human physiology and anatomy. Among these imaging modalities, USI is popular and widely applied in medicine because it is non-invasive, fast, cost-effective, and safe. In addition, the technique allows investigation of volume information in real-time[1]. These advantages have captured the attention of scholars to develop sophisticated techniques to enhance ultrasonograms, which are images recorded through USI.

Despite the merits, ultrasound images suffer from speckle noise that degrades their qualities, a consequence that may limit their usefulness. Speckle noise occurs when the back scattered echoes with spatial resolution less than that of the USI system interfere constructively or destructively, hence forming bright and dark spots which appear as granular structures on the reconstructed image[2]-[5]. Speckle patterns depict a form of multiplicative noise, and depends on the imaged tissue structures and various imaging parameters. This noise type reduces image contrast, limits detection of low-contrast lesions, introduces false structures and boundaries on the image, and also 
Signal \& Image Processing : An International Journal (SIPIJ) Vol.8, No.4, August 2017

degrades speed and accuracy of ultrasound imaging processing tasks such as edge detection, segmentation, registration, and volume rendering[6], [7]. One approach to improve quality of ultrasound images is to suppress speckle noise without affecting important features and texture of the image. Therefore, previous studies have attempted to address the challenges by proposing restoration and enhancement methods [1], [8]-[14]. And, the classical noise-suppressing frameworks for multilicative noise removal are total variation [15]-[23] wavelet [24]-[31] and linear/nonlinear diffusion [9], [32]-[35]. Some scholars have applied statistical theories to establish frameworks for noise estimation, and these approaches may promote further understanding on the denoising of ultrasonograms [36]-[38]. This work focuses on two frameworks, namely total variation (TV) and nonlinear diffusion, which have demonstrated higher performances in recovering critical image features. For the nonlinear diffusion framework, we have modified the Perona-Malik model [35] that has received considerable attention by scholars for its ability to restore high quality images. Contrary to several other approaches, we want to perform two tasks simultaneously: noise removal and preservation of useful image features (edges, contours, and lines). To this end, we have established a parametrized hybrid model that links TV and nonlinear diffusion frameworks to generate superior denoising results.

TV, pioneered by Rudin-Osher-Fatemi and hence the name ROF model[22] is based on the principle that the total variation of signals with excessive and possibly spurious details is high. Equivalently, TV formulation states that the integral of the absolute gradient of a noisy signal is high. Thus, minimizing the integral translates to reducing noise in the image. Rudin et al. used a constrained iterative optimization algorithm with Lagrange multipliers that impose the constraints on the total variation of the image. The authors applied gradient projection methods to obtain the solution. The TV minimization problem is defined by

$$
\underset{u \in B V \Omega}{\operatorname{argmin}}\left\{\left\|u_{B V}\right\|+\frac{\lambda}{2}\|u-f\|_{L^{2}(\Omega)}^{2}\right\}
$$

where $u$ and $f$ are the original and noisy images, $\mathrm{BV}(\Omega)$ denotes the bounded-variation space of functions, $\left\|u_{\mathrm{BV}}\right\|$ is the $\mathrm{BV}$ semi-norm, also known as total variation (TV) of $u$ given by $\left\|u_{\mathrm{BV}}\right\|=\int_{\Omega}|\nabla u|$, and $\lambda$ is the regularization parameter.

The Perona-Malik nonlinear diffusion model, originally conceived by Perona and Malik in 1990 and hence the name PM model, ensures that the diffusion process is conducted depending upon the local properties of an image. More specifically, the PM model encourages stronger denoising in homogeneous regions, which perceptually contain higher levels of noise, and weaker denoising near edges. This discriminatory behavior of the PM model helps to simultaneously suppress noise and to protect important image structures. To achieve their goal, Perona and Malik proposed a nonlinear partial diffusion equation that uses an inhomogeneous diffusivity coefficient. The idea behind the PM model is to modify the classical diffusion equation by adding the diffusivity coefficient that depends on space activity in a given part of an image, measured by the norm of the local image gradient. The Perona-Malik problem formulation with a Neumann boundary condition is given by

$$
\frac{\partial u}{\partial t}=\operatorname{div}\left(g\left(|\nabla u|^{2}\right) \nabla u\right) \quad(x, t) \in \Omega \times(0, T),
$$

where $x$ and $t$ represent space and time, respectively, $T$ is the total time, and $g$ defines the diffusivity coefficient. The authors proposed two definitions of $g$, namely 
Signal \& Image Processing : An International Journal (SIPIJ) Vol.8, No.4, August 2017

$$
g\left(|\nabla u|^{2}\right)=\left(\frac{1}{1+\left(\frac{|\nabla u|}{K}\right)^{2}}\right)
$$

and

$$
g\left(|\nabla u|^{2}\right)=\exp \left(-\left(\frac{|\nabla u|}{K}\right)^{2}\right)
$$

with $K$ denoting the shape-defining constant. The Perona-Malik model, however, suffers from staircasing problems and tends to add speckles into the evolving solutions [39], [40].

Our goal is to address the problems inherent in the TV and PM models. The proposed hybrid model gives a proper compromise between the models by ensuring that the regularization process produces results that are free from staircasing and speckle noise issues. Combination of TV and PM models has been achieved through parametrization that are designed to eliminate problematic portions of the corresponding energy functionals. Also, because we are dealing with multiplicative noise in ultrasonograms, we have added a log-based regularization prior into our framework. Extensive range of experiments have been conducted to demonstrate that our framework produces satisfactory results that are better than those of the classical models. Probably the most intriguing characteristic of our model is stability: results show that even at larger number of iterations the hybrid framework generates appealing results, an observation that eliminates a concern on setting the iteration limits or establishing the convergence criteria.

\section{HYBRID DIFFUSION-STEERED MODEL}

\subsection{Problem formulation}

Noting weaknesses of the classical PM model, we have established a hybrid diffusion framework that integrates two energy functionals, namely PM [35] and TV[22]. The framework is carefully designed to ensure that only convex portion of the PM potential is preferred. Therefore, additional parameters,

$$
\alpha= \begin{cases}0 & |\nabla \mathrm{u}|<\mathrm{H} \\ 1 & |\nabla \mathrm{u}| \geq \mathrm{H}\end{cases}
$$

and $\beta=\bar{\alpha}$, with $H$ denoting the shape-defining constant of the PM model, have been encapsulated into the framework to provide an automatic interplay between the two models. The values of $\alpha, \beta \in[0,1]$ assume only two states and are updated automatically within the iterative system to maintain stability and convexity of the framework.Hence, the proposed hybrid energy functional is given by

$$
\min _{u}\left\{E(s=|\nabla u|)=\alpha G s+\beta \frac{H^{2}}{2} \log \left(1+\left(\frac{s}{H}\right)^{2}\right)-\lambda\left(\log u+\frac{f}{u}\right)\right\}
$$

where $G$ is the gradient thresholding parameter for the TV model, $\lambda$ defines the regularization constant that refines a trade-off between $u$ and the noisy image, $f$. Equation 5 takes advantages from both TV and PM-the PM poor-behaving portions ( $s>H$ and $s<H$ ) are complimented by the TV well-behaving portions, a setting that may guarantee promising solutions. The last term of 5 defines a prior potential to suppress multiplicative noise[21]. 
Signal \& Image Processing : An International Journal (SIPIJ) Vol.8, No.4, August 2017

Solving (5) using the Euler-Lagrange method, and integrating the result into the time-dependent system, we get the evolution equation

$$
\begin{gathered}
\frac{\partial u}{\partial t}=\nabla \cdot\left(\frac{\alpha}{\frac{|\nabla u|}{G}} \nabla u\right)+\nabla \cdot\left(\frac{\beta}{1+\left(\frac{|\nabla u|}{H}\right)^{2}} \nabla u\right)-\lambda\left(\frac{u-f}{u^{2}}\right) \\
(x, t) \in \Omega \times(0, T) \\
u(x, 0)=f, x \in \Omega \\
\frac{\partial u}{\partial \vec{n}}=0,(x, t) \in \partial \Omega \times(0, T)
\end{gathered}
$$

where $u$ and $f$ are defined on $\Omega, x$ represents space or spatial coordinate, and $T>0$ is the time consumed by the whole iteration process. The first and second terms on the right side of (6) represent TV and PM diffusion functions, respectively, and the last part of the equation represents the regularization term. During execution of (6), the formulation is adjusted accordingly to reject undesirable solutions.

\subsection{Numerical Implementation}

Our model was implemented using the four-point explicit numerical scheme because of its simplicity, reliability, and accuracy[41]. Let $\nabla_{W}, \nabla_{N}, \nabla_{S}$, and $\nabla_{E}$ represent image gradients in the West $(\mathrm{W})$, North(N), South(S), and East(E) directions of the scheme. Then, the discrete image gradients can be defined as

$$
\begin{gathered}
\nabla_{W} u_{i, j}=u_{i},{ }_{j-1}-u_{i, j}, \nabla_{N} u_{i, j}=u_{i-1},{ }_{j}-u_{i, j}, \\
\nabla_{S} u_{i, j}=u_{i+1},{ }_{j}-u_{i, j}, \text { and } \nabla_{E} u_{i, j}=u_{i},{ }_{j+1}-u_{i, j}
\end{gathered}
$$

for $0 \leq i \leq I$ and $0 \leq j \leq J ; I$ and $J$ are the numbers of rows and columns, respectively; and $i$ and $j$ are row and column positions, respectively. The corresponding discrete conduction coefficients are

$$
\begin{aligned}
& c N_{i, j}^{(n)}=\frac{\alpha}{\left(\frac{\left|\nabla_{N} u_{i, j}^{(n)}\right|}{G}\right)}+\frac{\beta}{1+\left(\frac{\left|\nabla_{N} u_{i, j}^{(n)}\right|}{H}\right)^{2}}, \\
& c S_{i, j}^{(n)}=\frac{\alpha}{\left(\frac{\left|\nabla_{S} u_{i, j}^{(n)}\right|}{G}\right)}+\frac{\beta}{1+\left(\frac{\left|\nabla_{S} u_{i, j}^{(n)}\right|}{H}\right)^{2}}, \\
& c W_{i, j}^{(n)}=\frac{\alpha}{\left(\frac{\left|\nabla_{W} u_{i, j}^{(n)}\right|}{G}\right)}+\frac{\beta}{1+\left(\frac{\left|\nabla_{W} u_{i, j}^{(n)}\right|}{H}\right)^{2}},
\end{aligned}
$$


Signal \& Image Processing : An International Journal (SIPIJ) Vol.8, No.4, August 2017

$$
\text { and } c E_{i, j}^{(n)}=\frac{\alpha}{\left(\frac{\left|\nabla_{E} u_{i, j}^{(n)}\right|}{G}\right)}+\frac{\beta}{1+\left(\frac{\left|\nabla_{E} u_{i, j}^{(n)}\right|}{H}\right)^{2}} \text {. }
$$

Therefore, the discretized divergence term can be defined as

$$
\operatorname{div}_{i, j}^{(n)}=c N_{i, j}^{(n)} \nabla_{N} u_{i, j}^{(n)}+c S_{i, j}^{(n)} \nabla_{S} u_{i, j}^{(n)}+c W_{i, j}^{(n)} \nabla_{W} u_{i, j}^{(n)}+c E_{i, j}^{(n)} \nabla_{E} u_{i, j}^{(n)}
$$

and the steepest descent equation is

$$
u_{i, j}^{n+1}=u_{i, j}^{n}+\tau\left(\operatorname{div}_{i, j}^{n}-\lambda^{(n)}\left(u_{i, j}^{n}-f_{i, j}^{n}\right) /\left(\left(u_{i, j}^{n}\right)^{2}+\varepsilon\right)\right),
$$

where

$$
u_{i, j}^{(0)}=f_{i, j}=f(i h, j h), u_{i, 0}^{(n)}=u_{i, 1}^{(n)}, u_{0, j}^{(n)}=u_{1, j}^{(n)}, u_{I, i}^{(n)}=u_{I-1, i}^{(n)} \text {, and } u_{i, J}^{(n)}=u_{i, J-1}^{(n)}
$$

$h$ is the grid step size, $\tau$ is the time step, $\lambda$ is the regularization constant, and $\varepsilon>0$ defines a small stabilizing constant. Figure 1 shows the flow chart that we used to implement our formulation.

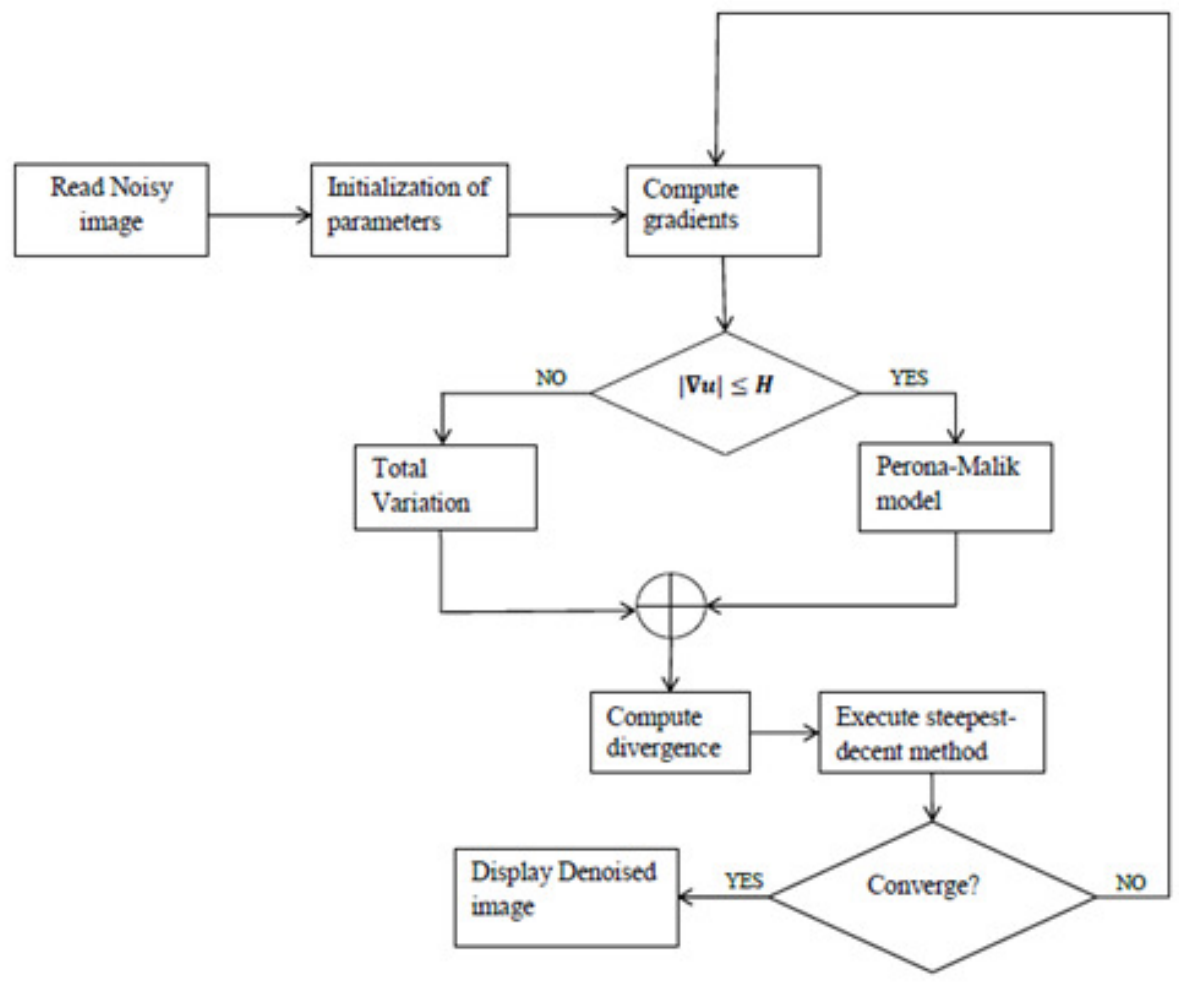

Figure 1: Flow diagram of the proposed framework.

\section{EXPERIMENTS AND EVALUATION METRICS}

\subsection{Experimental settings}

We conducted several experiments to test the performance of our model against other denoising methods, namely PM [35] Guo[39], Total variation [22] and Charbonnier [24]. In the first experiment, speckle (multiplicative) noise of density 0.04 was added into two different synthetic images, each $300 \times 300$ in size, namely "Geometry" and "Squares". Then, the methods were 
Signal \& Image Processing : An International Journal (SIPIJ) Vol.8, No.4, August 2017

applied on the noisy images to recover their original versions. Afterwards, we computed the corresponding error maps for each method. Next, objective quality metrics, namely PSNR and SSIM, were used to evaluate the restoration results for each method. The aspect of edge recovery was tested through image profiles: for each restored image, a line graph through intensity values along a specific row (arbitrarily taken half way on the vertical dimension) was drawn. These profiles were compared against those of the original and noisy images.

The second experiment involved real images of common carotic arteries (CCAs) corrupted by multiplicative noises. These CCAs were imaged from different patients. Then, the noise removal methods were applied to the images in an attempt to restore their (unknown) original versions. Because of a lack of the ground-truth images, comparisons on the results generated from various methods were done subjectively through visual assessments: an appealing image contains more details and holds semantically important features (edges, contours, and lines).

In all experiments, parameters of the proposed model were fixed: $\lambda=0.05, d t=0.15$, $G=\sqrt{1.3}, \quad H=1$, and $\varepsilon=1 \times 10^{-5}$. Simulation was done using MATLAB R2016b. Implementation codes of our framework have been shared in the MatlabCentralpublic repository ${ }^{1}$.

\subsection{Performance metrics}

Principally, two different approaches, namely subjective and objective, exist to assess quality of an image. The former approach can be achieved through questionnaires, in which case users apply their cognitive abilities to rate the visual attractiveness of the images. The approach, despite its wide acceptance by the ordinary community, is non-standard and tends to be expensive, inconvenient, and time consuming[42]. Objective image quality assessment involves development of quantitative measures that can automate the process of determining an image quality. The present work quantifies image qualities using PSNR (peak signal to noise ratio)[42] and SSIM (structural similarity) [43].

PSNR computes signal strength in the image relative to noise, and is defined by the equation

$$
\mathrm{PSNR}=20 \log _{10}\left(\frac{\mathrm{MAX}_{f}}{\sqrt{\mathrm{MSE}}}\right)
$$

where $\operatorname{MAX}_{f}$ is the maximum signal value in $f$ and MSE is the mean squared error. Stronger signals contain higher values of PSNR, and vice versa. The metric has, however, been criticized by scholars that it fails to mimic the human visual system [Wang et al.(2004)Wang, Bovik, Sheikh, and Simoncelli]. Consequently, Wang et al. proposed SSIM to address the problem. This quality index is defined as

$$
\operatorname{SSIM}=\frac{\left(2 \mu_{u} \mu_{f}+C_{1}\right)\left(2 \sigma_{u f}+C_{2}\right)}{\left(\mu_{u}^{2}+\mu_{f}^{2}+C_{1}\right)\left(\sigma_{u}^{2}+\sigma_{f}^{2}+C_{2}\right)},
$$

where $\mu_{u}$ and $\mu_{f}$ are the averages of $u$ and $f$, respectively; $\sigma_{u f}$ is the covariance of $u$ and $f ; \sigma_{u}$ and $\sigma_{f}$ are the standard deviations of $u$ and $f$, respectively; and $C_{1}$ and $C_{2}$ are stabilizing constants. SSIM, which ranges between 0 and 1 , is higher for a visually high-quality image, and vice versa.

\footnotetext{
${ }^{1}$ https://de.mathworks.com/matlabcentral/fileexchange/63681-hybrid-diffusion-steered-model-for-suppressi ng-multiplicative-noise-in-ultrasonograms
} 
Signal \& Image Processing : An International Journal (SIPIJ) Vol.8, No.4, August 2017

\section{RESULTS AND DISCUSSION}

Visual results from synthetic images demonstrate that the proposed method suppresses noise more effectively without affecting image features (Figures 2 and 3). Compared with the classical methods, our approach produces images with lower visible errors. Investigating profile maps, the proposed method shows that it generates sharper curves that are closer to the original ones (Figure 4). Hence, we infer that our approach can produce plausible edges. Applying the denoising methods on real ultrasound images (CCA images), we can observe that our method outperforms, as depicted by (Figures 7 and 8). The classical methods, on the contrary, generate unwanted artifacts. The restored CCA images by our method are more detailed, an observation that can assist doctors to provide more accurate and appropriate treatments to patients.

Quantitative results show that, in several cases, the proposed approach achieves higher values of PSNR and MSSIM (Table 1). Additionally, the approach generates PSNR and MSSIM curves that remain fairly uniform after attaining the peak values, which are higher than those obtained from the traditional methods (Figures 5 and 6). This observation indicates that our framework guarantees stability and may be applied with little restrictions on the number of iterations. Compelling results produced by our framework can be attributed to the well-designed energy functional. Also, our hybrid model is superior because it integrates the features of both total variation and Perona-Malik diffusion models, which complement one another to give enhanced mathematical properties.

Table 1: PSNR (peak-signal-to-noise-ratio) and SSIM (structural similarity) of images generated by different methods.

\begin{tabular}{lcc|cc}
\hline \multirow{2}{*}{ Method } & \multicolumn{2}{c|}{ PSNR } & SSIM & \\
\cline { 2 - 5 } & Geometry & Squares & Geometry & Squares \\
\hline Guo & 27.45 & 26.86 & 0.8752 & 0.9298 \\
Charbonnier & 29.82 & 29.95 & 0.9415 & 0.9781 \\
TV & 30.28 & 30.37 & 0.9720 & 0.9880 \\
PM & 31.43 & 30.41 & 0.9599 & 0.9646 \\
Proposed model & $\mathbf{3 1 . 6 1}$ & $\mathbf{3 0 . 6 4}$ & $\mathbf{0 . 9 8 0 5}$ & $\mathbf{0 . 9 9 1 9}$ \\
\hline
\end{tabular}
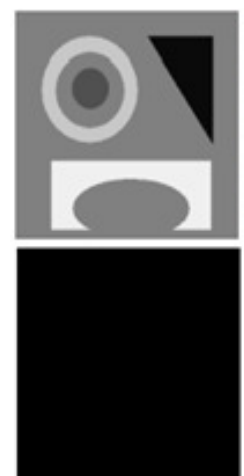

(a) Original
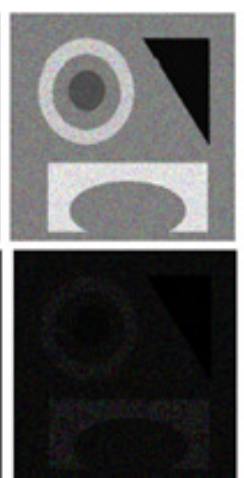

(b) Noisy
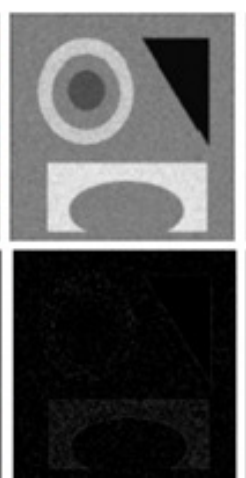

(c) Guo
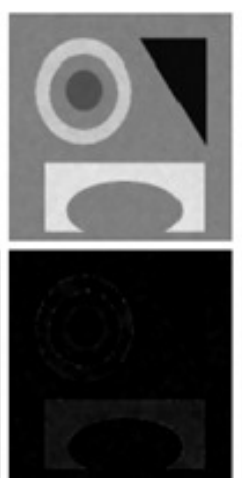

(d) PM

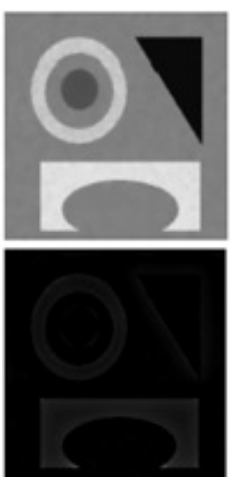

(e) TV

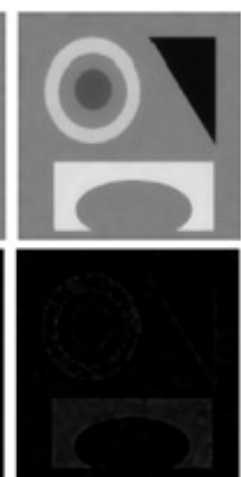

(f) Ours

Figure 2: Different denoising methods applied on the noisy "Geometry" synthetic image: first row, denoising results; and second row, corresponding error maps. PM, Perona-Malik; and TV, Total variation. 
Signal \& Image Processing : An International Journal (SIPIJ) Vol.8, No.4, August 2017

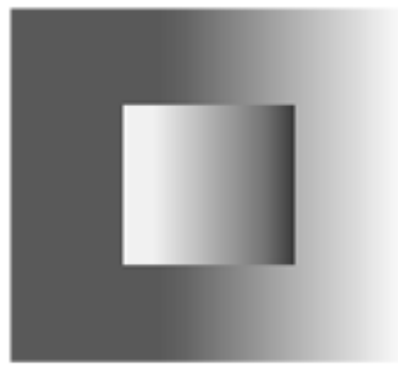

(a) Original

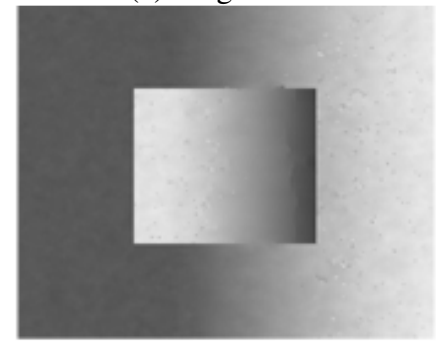

(d) PM

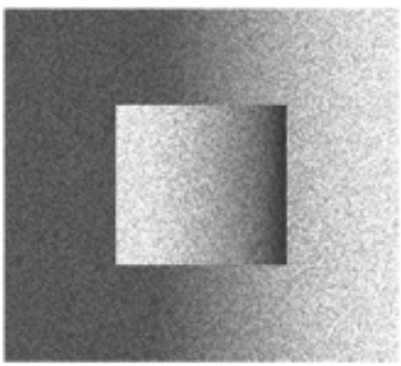

(b) Noisy

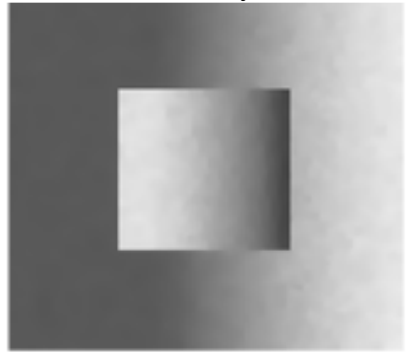

(e) TV

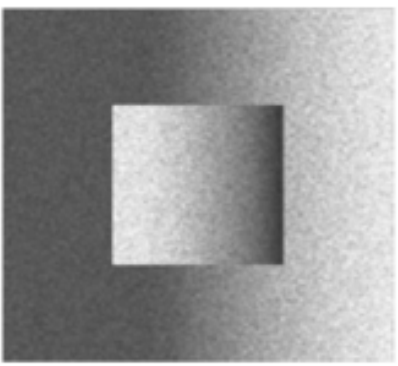

(c) Guo

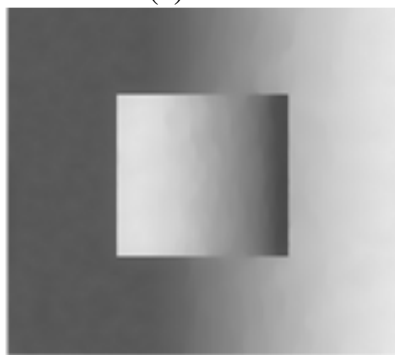

(f) Ours

Figure 3: Different denoising methods applied on the noisy "Squares" synthetic images

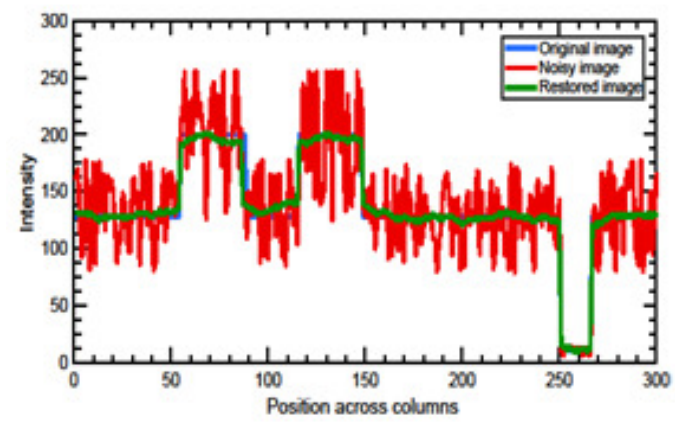

(a) Charbonnier

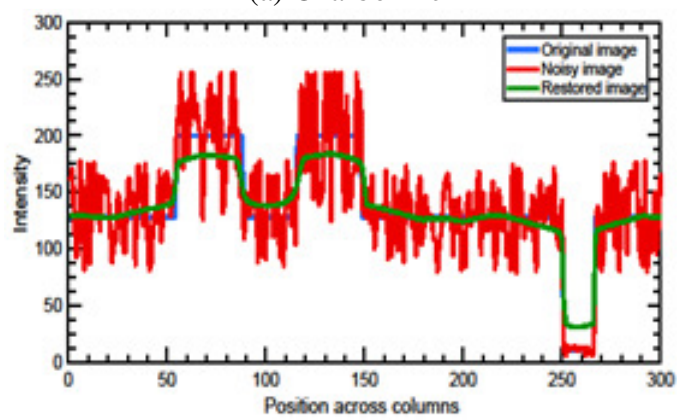

(c) Total variation

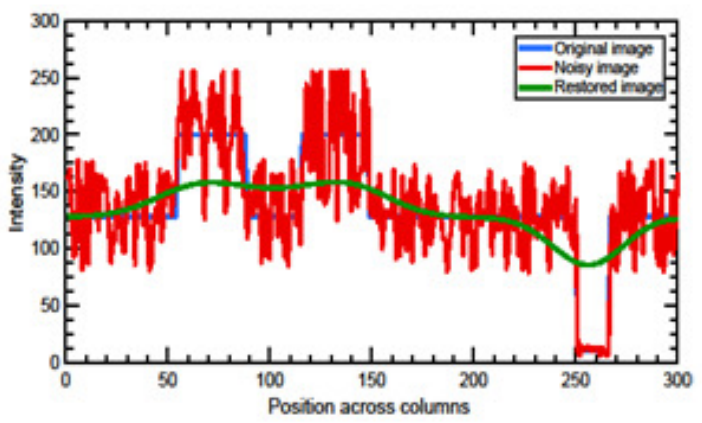

(b) Perona-Malik

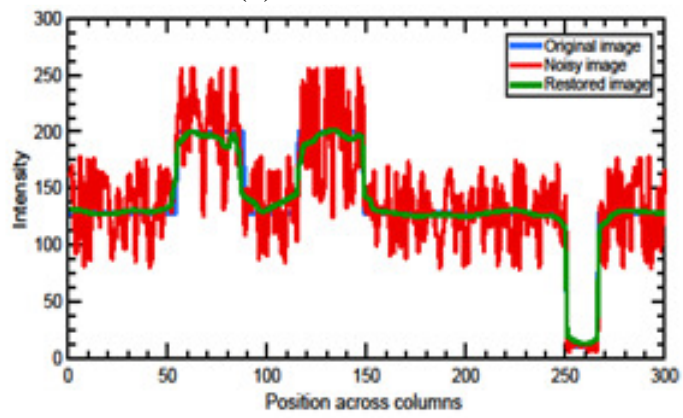

(d) Ours

Figure 4: Intensity image profiles of different denoising methods. 
Signal \& Image Processing : An International Journal (SIPIJ) Vol.8, No.4, August 2017
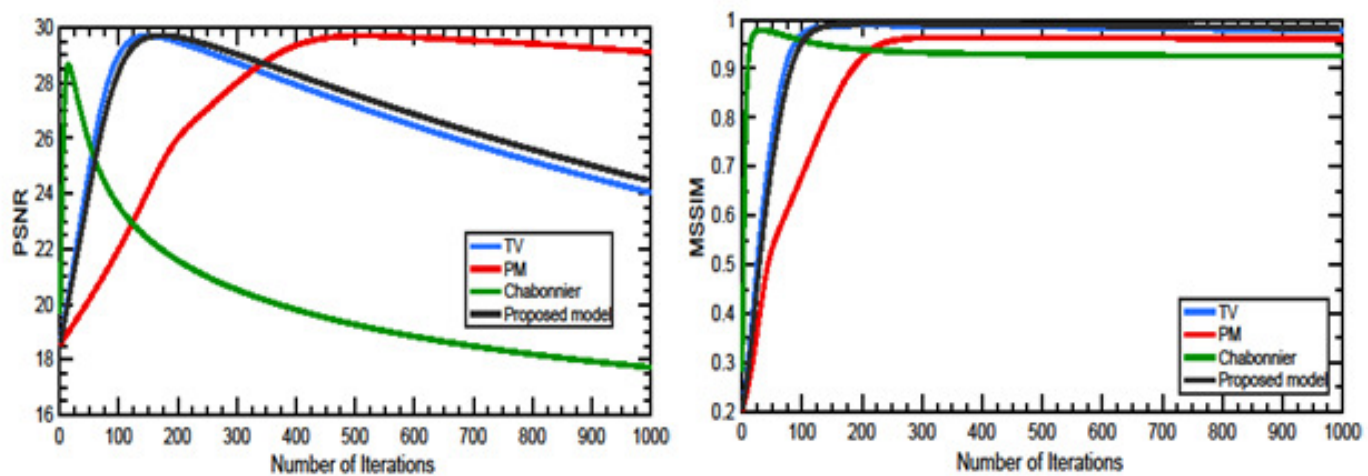

Figure 5: Peak-signal-to-noise-ratio (PSNR) and structural similarity (SSIM) of a "Geometry" image versus number of iterations.
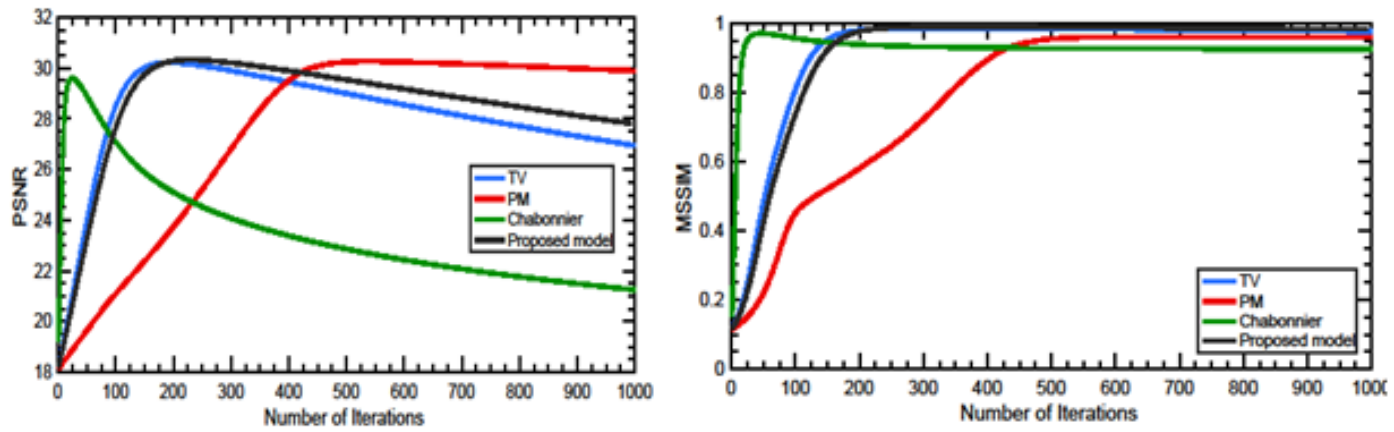

Figure 6: Peak-signal-to-noise-ratio(PSNR) and structural similarity (SSIM) of a "Squares"image versus number of iterations.

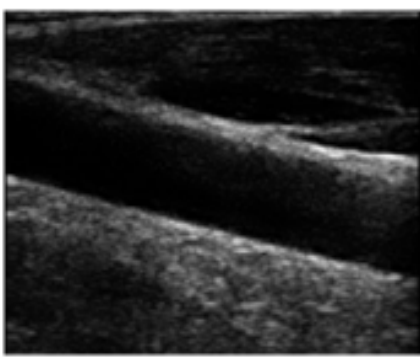

(a) Noisy

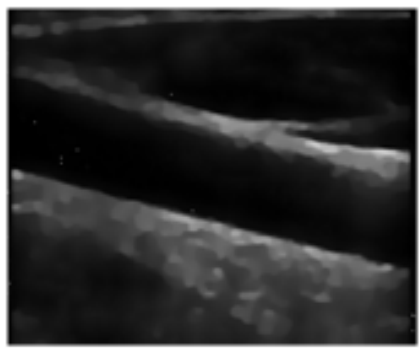

(d) TV

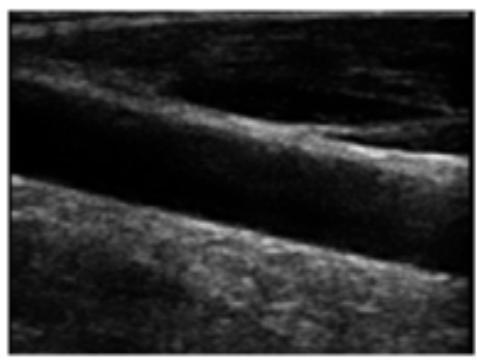

(b) Guo

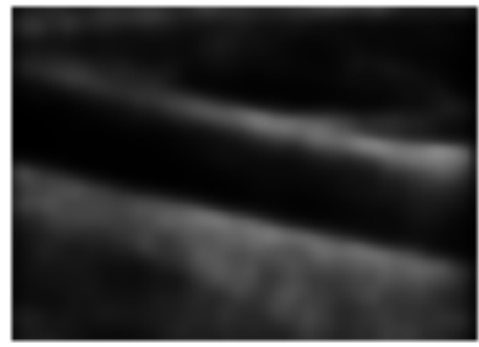

(e) Charbonneir

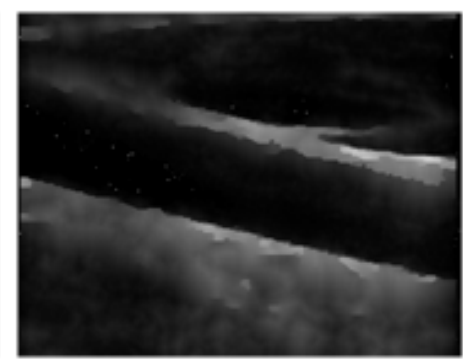

(c) PM

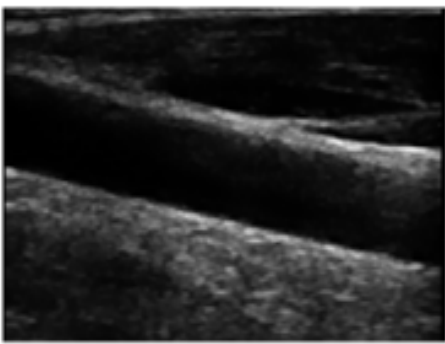

(f) Ours

Figure 7: Different denoising methods applied on a noisy common carotid artery (CCA) of patient \#1. 
Signal \& Image Processing : An International Journal (SIPIJ) Vol.8, No.4, August 2017

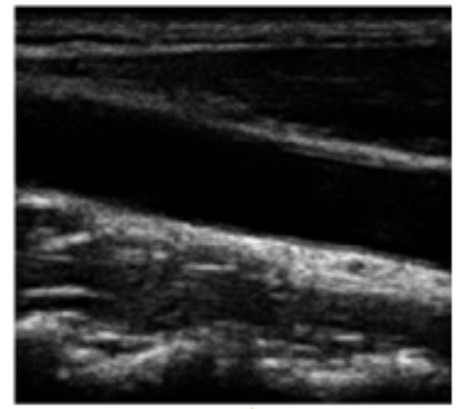

(a) Noisy

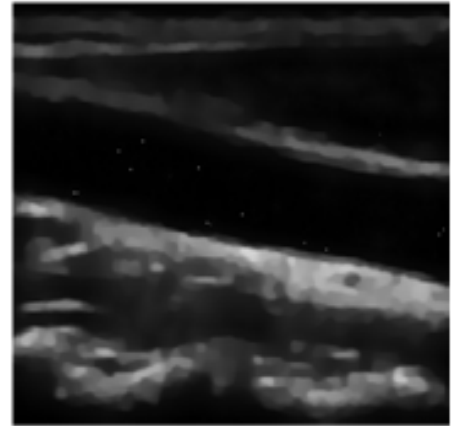

(d) TV

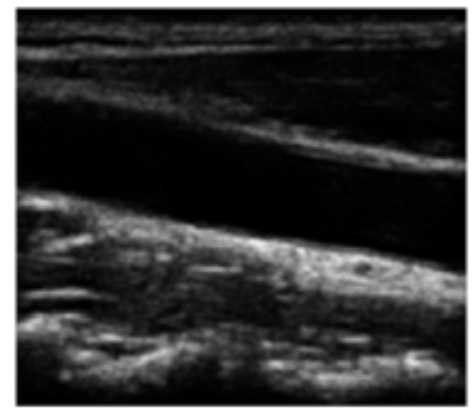

(b) Guo

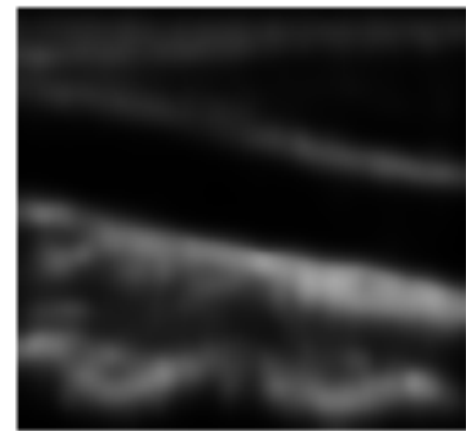

(e) Chabonneir

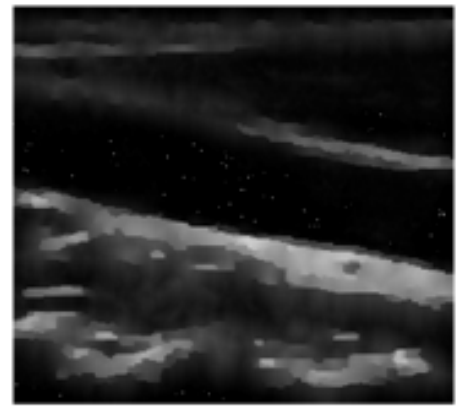

(c) PM

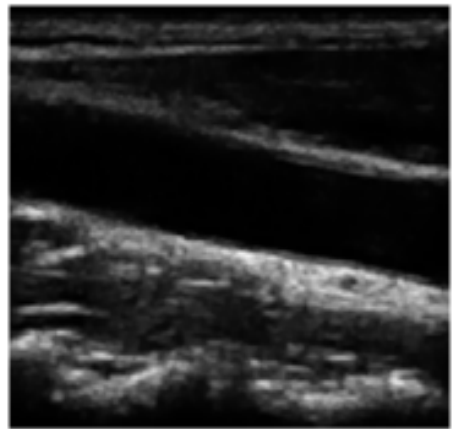

(f) Ours

Figure 8: Different denoising methods applied on a noisy common carotid artery (CCA) of patient \#2

\section{CONCLUSIONS}

The current work has proposed a hybrid diffusion-steered framework to suppress noise in ultrasound images. Our method combines denoising properties of TV and PM models. We have replaced the non-convex portion of the PM energy functional by the TV potential, a strategy that guarantees favorable solutions. Quantitative and subjective results demonstrate that our denoising framework outperforms, producing higher PSNR and MSSIM values compared with those from some classical methods. Also, our approach shows satisfactory results when applied to actual ultrasonograms, an evidence that the framework may suit clinical settings. In future, we intend to embed the proposed algorithm into real devices and deploy the system into hospitals to assist doctors in analysis and interpretation of ultrasound images.

\section{ACKNOWLEDGEMENTS}

This work was supported by DAAD (Deutscher Akademischer Austauschdienst) organization under the grant numbers 57191505 and 57349843 . Also, we would like to give our sincere gratitude to Prof. Gitta Domik (PhD) for her invaluable comments and suggestions to improve the quality of our work. Equally important, we thank the University of Paderborn and Nelson Mandela African Institute of Science and Technology for their social and academic support that impacted our study.

\section{Competing interests}

The authors declare that they have no competing interests to disclose. 
Signal \& Image Processing : An International Journal (SIPIJ) Vol.8, No.4, August 2017

\section{REFERENCES}

[1] O. V Michailovich and A. Tannenbaum, "Despeckling of medical ultrasound images.," IEEE Trans. Ultrason. Ferroelectr. Freq. Control, vol. 53, no. 1, pp. 64-78, Jan. 2006.

[2] J. W. Goodman, "Statistical properties of laser speckle patterns," in Laser Speckle and Related Phenomena, Berlin, Heidelberg: Springer Berlin Heidelberg, 1975, pp. 9-75.

[3] R. F. Wagner, S. W. Smith, J. M. Sandrik, and H. Lopez, "Statistics of Speckle in Ultrasound B-Scans," IEEE Trans. Sonics Ultrason., vol. 30, no. 3, pp. 156-163, 1983.

[4] V. Dutt, "Statistical analysis of ultrasound echo envelope," Ultrasound Res. Lab., p. 181, 1995.

[5] A. K. Boyat and B. K. Joshi, "A Review Paper: Noise Models in Digital Image Processing," Annu. Rev. Astron. Astrophys., vol. 43, no. 1, pp. 139-194, 2015.

[6] T. C. Aysal and K. E. Barner, "Rayleigh-maximum-likelihood filtering for speckle reduction of ultrasound images,” IEEE Trans. Med. Imaging, vol. 26, no. 5, pp. 712-727, 2007.

[7] A. Kaur, P. Sukhjeet, and K. Ranade, "Review of Speckle Noise Reduction Techniques for Ultrasound Imaging," no. 9, pp. 29-35, 2016.

[8] V. Bhateja, K. Rastogi, A. Verma, and C. Malhotra, "A non-iterative adaptive median filter for image denoising,” 2014 Int. Conf. Signal Process. Integr. Networks, no. February, pp. 113-118, 2014.

[9] G. Ramos-Llordén, G. Vegas-Sánchez-Ferrero, M. Martin-Fernandez, C. Alberola-López, and S. Aja-Fernández, "Anisotropic diffusion filter with memory based on speckle statistics for ultrasound images,” IEEE Trans. Image Process., vol. 24, no. 1, pp. 345-358, 2015.

[10] M. Kang, M. Kang, and M. Jung, "Total Generalized Variation Based Denoising Models for Ultrasound Images,” J. Sci. Comput., vol. 72, no. 1, pp. 172-197, Jul. 2017.

[11] Y. Hao, J. Xu, F. Zhang, and X. Zhang, "Multiplicative Noise Removal Based on the Linear Alternating Direction Method for a Hybrid Variational Model," vol. 2017, 2017.

[12] K. Singh, S. K. Ranade, and C. Singh, "Computer Methods and Programs in Biomedicine A hybrid algorithm for speckle noise reduction of ultrasound images," vol. 148, pp. 55-69, 2017.

[13] F. Baselice, "ULTRASOUND IMAGE DESPECKLING BASED ON STATISTICAL SIMILARITY," Ultrasound Med. Biol., pp. 1-14, 2017.

[14] J. Jaybhay and Rajveer Shastri, "A Study of Speckle Noise Reduction Filters," Signal Image Process. An Int. J., vol. 6, no. 3, pp. 71-80, 2015.

[15] J. Dong, Z. Han, Y. Zhao, W. Wang, A. Prochazka, and J. Chambers, "Sparse Analysis Model Based Multiplicative Noise Removal with Enhanced Regularization," Signal Processing, vol. 137, pp. 160-176, 2017.

[16] Y. Wu and X. Feng, "Speckle Noise Reduction via Nonconvex High Total Variation Approach," Math. Probl. Eng., vol. 2015, p. 11, 2015.

[17] M. Afonso and J. M. Sanches, "Image reconstruction under multiplicative speckle noise using total variation," Neurocomputing, vol. 150, no. Part A, pp. 200-213, 2015.

[18] M. G. Shama, T. Z. Huang, J. Liu, and S. Wang, "A convex total generalized variation regularized model for multiplicative noise and blur removal," Appl. Math. Comput., vol. 276, no. March, pp. 109-121, 2016. 
Signal \& Image Processing : An International Journal (SIPIJ) Vol.8, No.4, August 2017

[19] M. Hacini, F. Hachouf, and K. Djemal, "A new speckle filtering method for ultrasound images based on a weighted multiplicative total variation," Signal Processing, vol. 103, pp. 214-229, 2014.

[20] X. Lv, J. Le, J. Huang, and L. Jun, “A Fast High-Order Total Variation Minimization Method for Multiplicative Noise Removal,” Math. Probl. Eng., vol. 2013, p. 13, 2013.

[21] J. Liu, T. Huang, Z. Xu, and X. Lv, "High-order total variation-based multiplicative noise removal with spatially adapted parameter selection,” J. Opt. Soc. Am. A, vol. 30, no. 10, p. 1956, 2013.

[22] L. I. Rudin, S. Osher, and E. Fatemi, "Nonlinear total variation based noise removal algorithm," Phys. D, vol. 60, no. 1-4, pp. 259-268, 1992.

[23] V. N. P. Raj and T. Venkateswarlu, "Denoising of Medical Images Using Total Variational Method," Signal Image Process. An Int. J., vol. 3, no. 2, pp. 131-142, 2012.

[24] P. Charbonnier, L. Blanc-Feraud, G. Aubert, and M. Barlaud, "Two deterministic half-quadratic regularization algorithms for computed imaging," in Image Processing,1994. IEEE International conference, 1994, no. 4, pp. 168-172.

[25] D. L. Donoho, “De-Noising by Soft-Thresholding," IEEE Trans. Inf. Theory, vol. 41, no. 3, pp. 613-627, 1995.

[26] S. G. Chang, B. Yu, and M. Vetterli, "Adaptive wavelet thresholding for image denoising and compression,” Image Process. IEEE Trans., vol. 9, no. 9, pp. 1532-1546, 2000.

[27] M. Mastriani, "New Wavelet-Based Superresolution Algorithm for Speckle Reduction in SAR Images," no. 1, pp. 944-951, 2008.

[28] B. a. Abrahim, Z. a. Mustafa, I. a. Yassine, N. Zayed, and Y. M. Kadah, "Hybrid Total Variation and Wavelet Thresholding Speckle Reduction for Medical Ultrasound Imaging," J. Med. Imaging Heal. Informatics, vol. 2, no. 2, pp. 114-124, 2012.

[29] J. Zhang, G. Lin, L. Wu, C. Wang, and Y. Cheng, "Wavelet and fast bilateral filter based de-speckling method for medical ultrasound images," Biomed. Signal Process. Control, vol. 18, pp. 1-10, 2015.

[30] S. Kour and B. Kaur, "Hybrid filter with wavelet denoising and anisotropic diffusion filter for image despeckling,” 2015 IEEE Int. Conf. Comput. Graph. Vis. Inf. Secur. CGVIS 2015, vol. 115, no. 19, pp. 17-21, 2016.

[31] T. Thomas, "Image Denoising Using Sure-Based Adaptive Thresholding In Directionlet Domain," Signal Image Process. An Int. J., vol. 3, no. 6, pp. 61-73, 2012.

[32] Z. Hu and J. Tang, "Cluster driven anisotropic diffusion for speckle reduction in ultrasound images," in 2016 IEEE International Conference on Image Processing (ICIP), 2016, pp. 2325-2329.

[33] J. Xu, Y. Jia, Z. Shi, and K. Pang, "An improved anisotropic diffusion filter with semi-adaptive threshold for edge preservation,” Signal Processing, vol. 119, pp. 80-91, 2016.

[34] B. Vikrant, S. Gopal, and S. Atul, "A Novel Weighted Diffusion Filtering Approach for Speckle Suppression in Ultrasound Images,” Adv. Intell. Syst. Comput., vol. 247, no. January 2014, 2014.

[35] P. Perona and J. Malik, "Scale-space and edge detection using anisotropic diffusion.," IEEE Trans. Pattern Anal. Mach. Intell., vol. 12, no. 12, pp. 629-639, 1990.

[36] M. Imani and U. M. Braga-Neto, "Maximum-Likelihood Adaptive Filter for Partially Observed Boolean Dynamical Systems,” IEEE Trans. Signal Process., vol. 65, no. 2, pp. 359-371, 2017. 
Signal \& Image Processing : An International Journal (SIPIJ) Vol.8, No.4, August 2017

[37] A. Doucet, S. Godsill, and C. Andrieu, "On sequential Monte Carlo sampling methods for Bayesian filtering,” Stat. Comput., pp. 197-208, 2000.

[38] M. Imani and U. Braga-Neto, "Particle Filters for Paertially-Observed Boolean Dynamical Systems," IEEE Trans. Signal Process., vol. PP, no. 99, p. 1, 2016.

[39] Z. Guo, J. Sun, D. Zhang, and B. Wu, “Adaptive Perona - Malik Model Based on the Variable Exponent for Image Denoising,” Image Process. IEEE Trans., vol. 21, no. 3, pp. 958-967, 2012.

[40] B. Wu, E. A. Ogada, J. Sun, and Z. Guo, “A Total Variation Model Based on the Strictly Convex Modification for Image Denoising,” Abstr. Appl. Anal., vol. 2014, p. 16, 2014.

[41] H. P. Langtangen, "Finite difference methods for diffusion processes," pp. 1-21, 2013.

[42] Z. Wang and A. C. Bovik, "Mean squared error: Lot it or leave it? A new look at signal fidelity measures," IEEE Signal Process. Mag., vol. 26, no. 1, pp. 98-117, 2009.

[43] Z. Wang, A. C. Bovik, H. R. Sheikh, and E. P. Simoncelli, "Image quality assessment: From error visibility to structural similarity,” IEEE Trans. Image Process., vol. 13, no. 4, pp. 600-612, 2004.

\section{AUTHOR}

Suzan Kessy is currently a master's student in Information and Communication Science and Engineering at Nelson Mandela African Institution of science and Technology (NM-AIST) specializing in Electronics and telecommunication engineering. She received BSc (Hons) degree in Electronics science and Communication at University of Dar-es-salaam in November 2014. Her interest includes Electronics, Image processing, computer graphics and Data visualization

Baraka Maiseli earned his Doctoral degree in control science and engineering from the Harbin Institute of Technology, PR China, in 2015. He has published several articles in referred journals. Also, Baraka has peer-reviewed a number of articles in international journals: IEEE Transactions in Industrial Electronics, Signal Processing, Neuro-computing, IET Biometrics, IET Control Theory \& Applications, Abstract and Applied Analysis, Digital Signal Processing, among others. Currently, Baraka is a lecturer at the University Of Dar-es-Salaam, Tanzania. He has been a postdoctoral fellow at the Harbin Institute of Technology since 2016.

Michael Kisangiri Received his Ph.D. degree in Telecommunication Engineering (2008) and Master of Science in Telecommunication Engineering (2002), both from Wrocław University of Technology - Poland. Since then he has been working as academician with several universities. Currently he works with Nelson Mandela African Institution of Science and Technology located in Arusha, Tanzania. His research interests include evolutionary computation in Telecommunication Networks, Antenna design and Triangular mesh modeling.
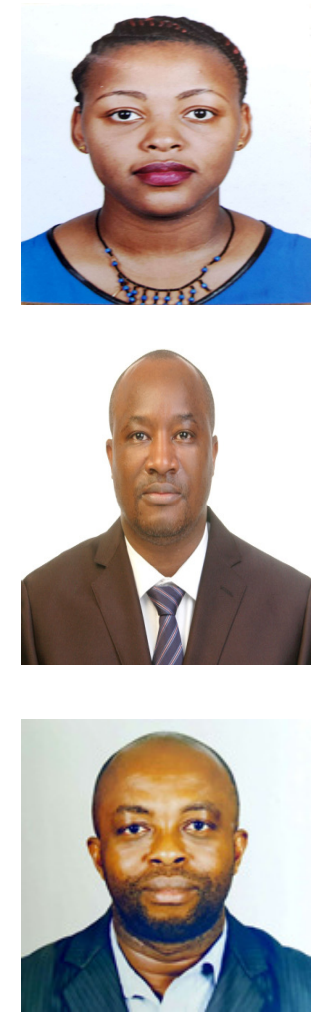